\title{
Low-loss wavelength-selective integrated waveguide coupler based on tilted Bragg gratings
}

\author{
Mathias J. Weisen ${ }^{1, *}$, Matthew T. Posner ${ }^{1}$, James C. Gates ${ }^{1}$, Corin B. E. Gawith ${ }^{1}$, Peter G. \\ R. SMITH ${ }^{1}$, ANd PETER HORAK ${ }^{1}$ \\ ${ }^{1}$ Optoelectronics Research Centre, University of Southampton, Southampton SO17 1BJ, United Kingdom \\ *Corresponding author: m.j.weisen@soton.ac.uk
}

Compiled May 22, 2019

\begin{abstract}
We propose and investigate numerically a new kind of integrated optical waveguide coupler. Two waveguides with tilted Bragg gratings couple selectively to a discrete counter-propagating cladding mode which acts as an intermediary to transfer power between the waveguides. This gives a highly versatile platform that can act as a low-loss coupler with arbitrary coupling ratio between 0 and $100 \%$, as a narrowbandwidth wavelength filter, and as a phase-selective switch that transmits two light waves of a fixed relative phase but reflects the orthogonal phase within a single device. ๑ 2019 Optical Society of America
\end{abstract}

http://dx.doi.org/10.1364/ao.XX.XXXXXX

\section{INTRODUCTION}

Directional couplers are a key component in planar photonics circuits as well as in optical fiber networks. Traditionally, coupling is achieved by bringing two waveguides close together in order to cause a spatial overlap between their modes [1]. Variations on this scheme utilize Bragg gratings to assist phase matched coupling between asymmetric waveguides [2], frustrate coupling at a particular frequency [3] or to form add-drop multiplexers [4]. Similar devices have also been realized in non-silica material platforms, e.g., grating assisted wavelength selective couplers [5] and wavelength division multiplexers [6, 7] have been demonstrated in silicon photonics.

By tilting the grating planes of a Bragg grating away from perpendicular to the propagation direction of the waveguides the full spatial degrees of freedom of light propagation in the device can be exploited, which opens up a host of novel device functionalities. Such tilted gratings work by not only phase matching modes in the forward propagation direction, but also matching the propagation constants in the transverse direction to achieve efficient coupling of waveguides to unbound radiation modes [8] or to higher order guided or cladding modes [9] in analogy to blazed diffraction gratings. While such tilted Bragg gratings offer significant potential benefits in efficiency and tunability of waveguide devices, only a few applications have been demonstrated so far, such as in spectrometry [10], waveguide division multiplexing [11], out of plane coupling to a Gaussian mode [12], and refractometry [13].

Here we investigate a new design of a directional coupler based on tilted Bragg gratings where the two waveguides sit on a common ridge structure cut into the substrate. The ridge structure only supports a limited number of discrete cladding modes, and thus by tailoring the grating parameters it is possible to design an optical coupler utilizing only a single cladding mode as intermediary between the waveguides. This allows for low-loss waveguide coupling with arbitrary coupling efficiency up to $100 \%$. By using a counter-propagating cladding mode the coupling becomes highly wavelength selective and the device acts as a narrowband coupler and/or wavelength filter. We also show that the device can operate as a phase-sensitive switch: if light is coupled simultaneously into both waveguides, then depending on the relative phase of the two waves they can either be transmitted through the device by a grating induced transparency [14] or completely reflected.

Apart from applications as filters and wavelength division multiplexers in optical telecommunication systems, the wide range of potential functionalities and low loss of our device will make it a powerful component in quantum information networks. For example, it could enhance multiphoton quantum interference devices $[15,16]$ by allowing photon coupling between waveguides beyond nearest neighbors, or act as a narrow-band filter to separate entangled photons generated by frequency conversion from the strong pump field [17].

Fabrication of such devices is feasible using direct UV writing, a laser fabrication technique for the simultaneous definition of waveguides and gratings through the exposure of photosensitive silica layers to UV radiation [18]. It offers excellent phase stability and control of grating properties, low cost and low insertion loss [18, 19]. Polarizers [20] and polarizing waveguide to waveguide couplers [21] based on this technology were fabricated. However, these devices exploited a continuum of substrate modes as intermediaries to couple light between waveguides, which led to very low coupling efficiencies compared 
to those achievable if a discrete set of cladding modes is used [22-24].

This paper is structured as follows. In Sec. 2 we describe the geometry of the coupler and give an overview of the coupled mode theory we use to simulate the device. We then derive an analytical solution of the coupled mode model in the limit where only one cladding mode is coupled effectively to the waveguides in Sec. 3 and discuss grating induced transparency in Sec. 4. In Sec. 5 we show that the numerical and analytical models are consistent and we discuss some main properties of the device. In Sec. 6 we investigate the parameter dependence of the device operation bandwidth and in Sec. 7 its temperature sensitivity. Finally, we summarize and conclude our findings in Sec. 8.

\section{DEVICE GEOMETRY AND THEORETICAL DESCRIP- TION}

A cross section schematic of the proposed device is shown in Fig. 1(a). Using direct UV writing, two parallel single-mode channel waveguides are fabricated, simultaneously with two identical tilted Bragg gratings, into a photosensitive raised refractive index core layer that forms part of a ridge structure. The ridge can be created by physically micro-machining two trenches on either side using the same fabrication method as [13, 25]. The narrow core layer confines light to a single transverse mode in the vertical $(y)$ direction, while the ridge structure provides confinement in the $x$-direction and thus only allows for a finite number of discrete modes in the core layer with well separated modal effective indices. This structure therefore maximizes overlap and coupling between the waveguide modes and the cladding modes within this core layer. Fig. 1(b) shows a schematic of the refractive index profile in the $x$-direction inside the core layer and three of the modes guided in this core layer, calculated by a finite element method (FEM) using Comsol Multiphysics ${ }^{\circledR}$ for a core layer of thickness $2.0 \mu \mathrm{m}$ and a refractive index of 1.4468 and overclad and underclad layers with a refractive index of 1.4368 .

Our design features Bragg gratings with a short period and a small tilt angle in order to couple the waveguide modes to the backward propagating cladding modes of the ridge to allow for efficient coupling and short device lengths. The cladding modes are then used as an intermediary between the two waveguides as shown in Fig. 1(c). All light remains within the modal structure of the waveguides and the cladding and so, unlike in a system exploiting the continuum of substrate modes as intermediaries [21], there is in theory no loss of light from coupling to unbounded modes. There is also no need to bring the waveguides physically close together as in evanescent field couplers.

For clarity we use a simplified theoretical model of the device in the following. First, we only consider a two-dimensional model, i.e., we ignore the thin core layer that provides confinement in the $y$-direction and assume an infinitely extended structure in this direction instead. Second, we only consider the transverse electric (TE, or $y$-polarized) modes of the device. In this case the modes exhibit only one component of the electric field which satisfies the wave equation

$$
\frac{d^{2} \psi(x)}{d x^{2}}+k_{0}^{2}\left[n^{2}(x)-n_{z}^{2}\right] \psi(x)=0
$$

where $\psi(x)$ is the transverse profile of the mode, $n_{z}=\beta_{z} / k_{0}$ is the effective refractive index in the $z$-direction, $k_{0}$ is the free space wavenumber of the light and $\beta_{z}$ is the $z$-component of the propagation constant for this mode. $n(x)$ is the transverse
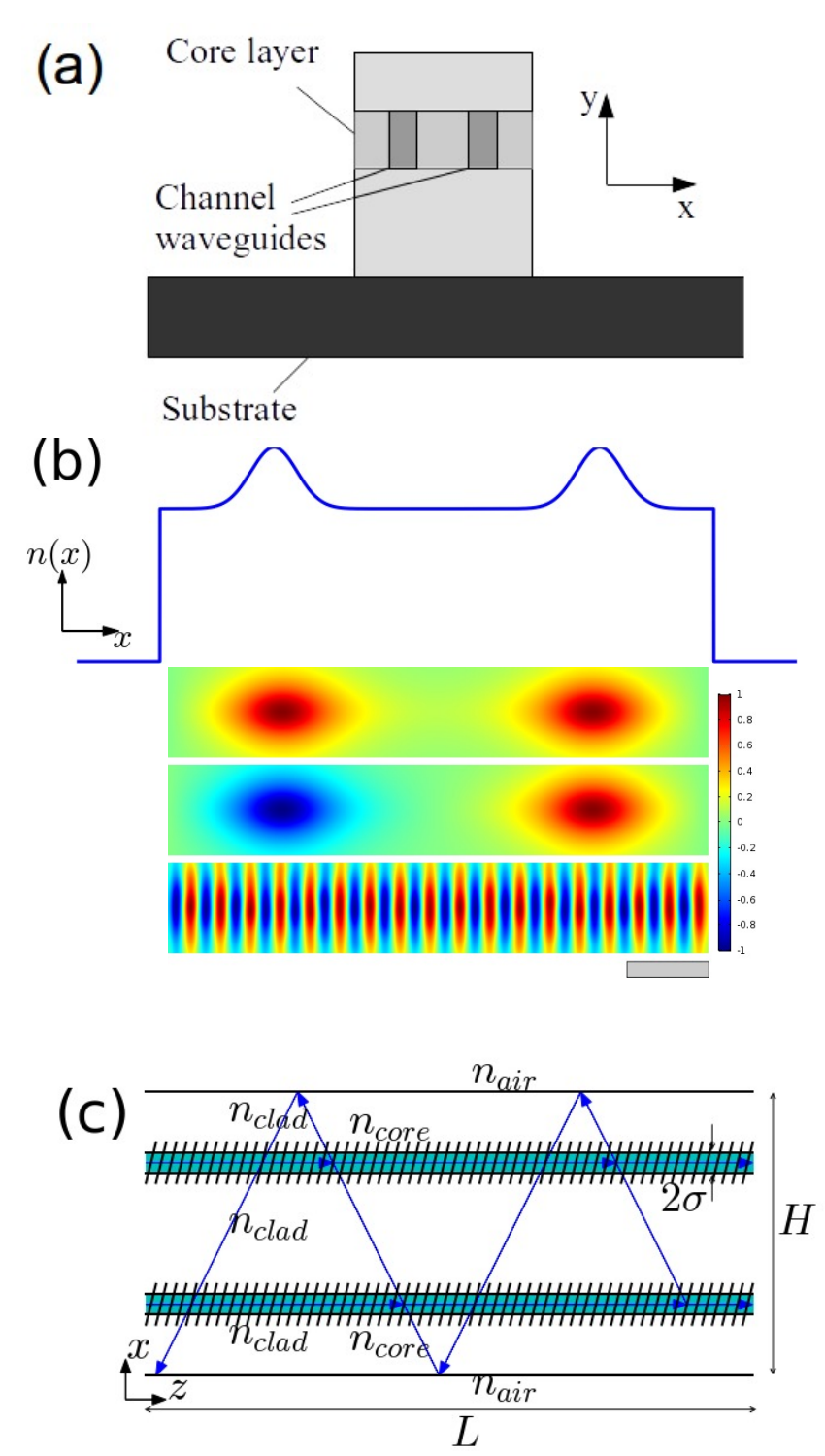

Fig. 1. (a) $2 \mathrm{D}$ cross section of the cladding-mode based waveguide coupler. (b) Schematic of the refractive index profile across the core layer and FEM calculations of the symmetric core mode, antisymmetric core mode, and a higher order cladding mode, all confined within the core layer. The grey scalebar corresponds to $5 \mu \mathrm{m}$. (c) Top view of the structure inside the core layer, where the waveguides are indicated in cyan and the tilted gratings by the diagonal grid lines, with definition of system parameters and ray diagram of coherently scattered light from the gratings. 
refractive index profile of the cladding and the waveguides and is defined as

$$
\begin{aligned}
& n(x)=n_{\text {clad }}+\Delta n \rho(x) \\
& \rho(x)=\exp \left(\frac{-\left(x-x_{0}\right)^{2}}{\sigma^{2}}\right)+\exp \left(\frac{-\left(x+x_{0}\right)^{2}}{\sigma^{2}}\right)
\end{aligned}
$$

inside the region $|x| \leq H / 2$ and $n(x)=1$ outside of it; $\rho(x)$ is the transverse profile of the waveguides. This defines two waveguides of refractive index contrast $\Delta n$ and of $1 / e$ width $\sigma$ that are placed at a distance of $\pm x_{0}$ from the center of the ridge. The ridge itself has a width of $H$ and a refractive index of $n_{\text {clad }}$. We choose $\Delta n$ to be sufficiently small such that the waveguides support only a single mode. The eigenmodes $\psi_{n}(x)$ of the wave equation (1) with the refractive index profile (2) are then calculated numerically using a finite differences method.

Without the use of Bragg gratings the waveguide modes would remain decoupled from each other. The gratings act as a perturbation to the original waveguide structure leading to coupling between the waveguide modes and the cladding modes. The perturbation from the Bragg gratings is defined by

$$
\delta n(x)=\Delta n_{g} \rho(x) \sin \left[K_{g}(z-x \tan \theta)\right]
$$

where $\Delta n_{g}$ is the refractive index modulation of the gratings, $K_{g}$ is the longitudinal wave vector of the grating, $\theta$ is the tilt angle, and the transverse profile $\rho(x)$ is the same as for the waveguides themselves, Eq. (3).

Applying standard coupled mode theory [19], we can then calculate the coupling coefficients between modes $\psi_{n}$ and $\psi_{m}$ of the device,

$$
\begin{aligned}
d_{m n+}= & \frac{k_{0}}{2 c \mu_{0}} \operatorname{sign}(m) \\
& \times \int n(x) \Delta n_{g} \cos \left(K_{t} x\right) \rho(x) \psi_{m}^{*}(x) \psi_{n}(x) d x
\end{aligned}
$$

and

$$
\begin{aligned}
d_{m n-}= & -\frac{k_{0}}{2 c \mu_{0}} \operatorname{sign}(m) \\
& \times \int n(x) \Delta n_{g} \sin \left(K_{t} x\right) \rho(x) \psi_{m}^{*}(x) \psi_{n}(x) d x
\end{aligned}
$$

where $c$ is the speed of light and $K_{t}=K_{g} \tan \theta$ is the transverse wavevector and we have introduced the notation that $m>0$ denotes forward propagating modes and $m<0$ backward propagating modes. The two components $d_{m n \pm}$ can be rewritten as an amplitude and phase,

$$
\begin{aligned}
& g_{m n}=\sqrt{d_{m n+}^{2}+d_{m n--}^{2}}, \\
& \phi_{m n}=\tan ^{-1}\left(\frac{d_{m n-}}{d_{m n+}}\right) .
\end{aligned}
$$

The dynamics of the electric field amplitudes $A_{m}(z)$ propagating in modes $m$ along the device are then given by the coupled mode equations [19]

$$
\begin{aligned}
\frac{d A_{m}(z)}{d z}= & -\frac{1}{2} \sum_{n}\left(e^{i\left[\left(\beta_{m}-\beta_{n}+K_{g}\right) z+\phi_{m n}\right]}\right. \\
& \left.-e^{i\left[\left(\beta_{m}-\beta_{n}-K_{g}\right) z-\phi_{m n}\right]}\right) A_{n}(z) g_{m n}
\end{aligned}
$$

where $\beta_{m}=\beta_{z}$ for mode $m$.
In this paper we assume short period Bragg gratings that couple only modes traveling in opposite directions. This allows us to greatly simplify the problem and only consider the two forward propagating waveguide modes and the backward propagating cladding modes. Eq. (9) can then be rewritten as

$$
\frac{d \mathcal{R}_{m}(z)}{d z}=i\left(\frac{K_{g}}{2}-\beta_{m}\right) \mathcal{R}_{m}(z)+\frac{1}{2} \sum \mathcal{R}_{-n}(z) g_{m n} e^{-i \phi_{m n}}
$$

where $\mathcal{R}_{m}(z)=A_{m} e^{i K_{g} z / 2}$ and $\mathcal{R}_{-m}(z)=A_{-m} e^{-i K_{g} z / 2}$.

Eq. (10) is then solved numerically as follows. First, the eigen solutions of this linear set of equations are found. Next, the boundary conditions are applied, which in our case are that light is launched at $z=0$ into only one waveguide, whereas the other waveguide is empty at $z=0$. Additionally, the backward propagating cladding modes are all empty at the output, $z=L$, where $L$ is the device length. These boundary conditions give a linear set of equations for the amplitudes of the eigen solutions of (10), which is solved easily and thus contains the full system dynamics along $z$. A full analytical solution in the special case where only a single cladding mode is involved is given below in Sec. 3.

We consider a cladding of refractive index $n_{\text {clad }}=1.4398$ and width $H=34 \mu \mathrm{m}$. Two waveguides of refractive index depth $\Delta n=0.01$ and width $\sigma=2.0 \mu \mathrm{m}$ are written at $x_{0}=10 \mu \mathrm{m}$ from the center of the device, and the light wavelength is $\lambda_{0}=$ $1.55 \mu \mathrm{m}$. To ensure accurate computation of modes, we use 4001 grid points in the ridge region including one at each interface and include $5 \mu \mathrm{m}$ of air on either side in the simulation. The finite differences method is computed with an accuracy level of 4 as defined in [26]. For these parameters, 46 waveguide and cladding modes have been found as shown in Fig. 2. We see that the two waveguide modes have near identical $n_{z}$ which implies that evanescent coupling between them is very weak. Higher order modes are spaced increasingly further apart, as expected for a step-index profile. In Fig. 3 we show the transverse mode profiles of the 0th, 1st and 35th modes which we mainly use in our discussions below.

In Fig. 4 we show the coupling coefficients of the cladding modes to the waveguide modes for different values of the transverse wave vector of the grating $K_{t}=K_{g} \tan \theta$. For $K_{t}=0$, i.e. with grating planes perpendicular to the waveguides, coupling is strongest to the low order modes which only have a small transverse propagation constant. Higher order modes, on the other hand, with a larger transverse propagation constant are not phase matched in the transverse direction and are therefore only weakly coupled. By introducing a tilt angle, i.e. increasing $K_{t}$, efficient coupling to increasingly higher order cladding modes is observed. Because of the finite physical width of the grating, its transverse index profile has Fourier components at wavenumbers other than $K_{t}$ which allows for coupling to a relatively broad range of cladding modes. This implies that the device is relatively robust to the fabrication tolerance of the tilt angle. Oscillations in the $K_{t}=0.0 \mu \mathrm{m}^{-1}$ curve in Fig. 4 are a result of changing overlap between the cladding mode and the waveguides. If a maximum (or minimum) of the electric field of a mode coincides with a waveguide, this leads to a larger (or smaller) coupling coefficient.

\section{ANALYTICAL SOLUTION}

If the cladding modes have sufficiently different values of $n_{z}$, the grating properties can be chosen such that only a single (backward propagating) cladding mode is phase matched to 


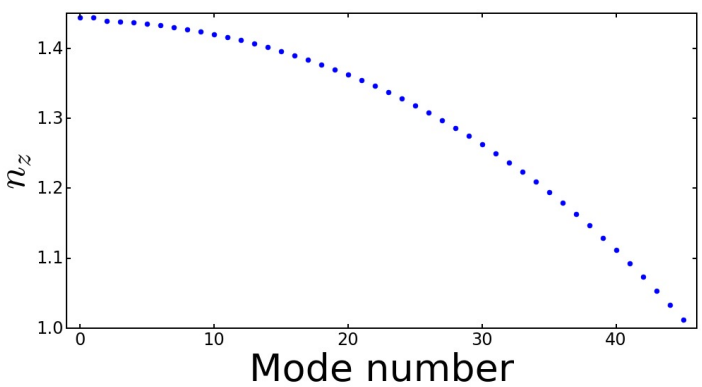

Fig. 2. Value of $n_{z}$ against mode number with $\Delta n=0.01$, $\sigma=2.0 \mu \mathrm{m}, x_{0}=10 \mu \mathrm{m}$ and $H=34 \mu \mathrm{m}$.

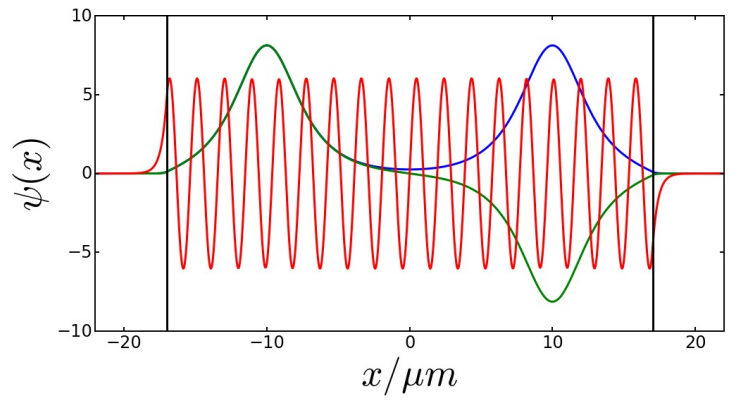

Fig. 3. Transverse profile of modes 0 (blue), 1 (green) and 35 (red) which are the symmetric and anti-symmetric core modes and a cladding mode, respectively. The vertical lines indicate the interface between the ridge structure and the surrounding air.

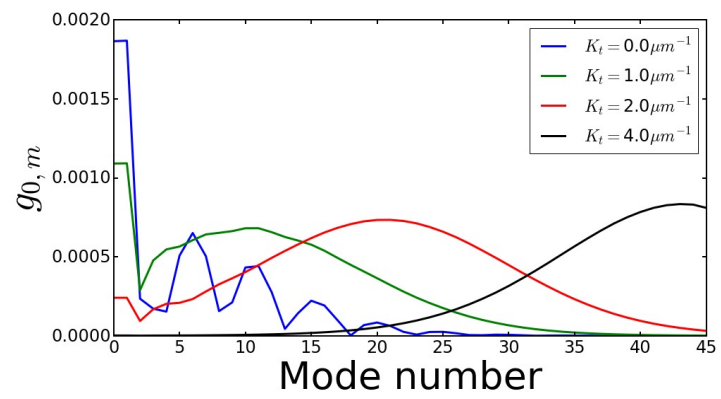

Fig. 4. Coupling coefficients of the waveguide modes to the cladding modes for different values of $K_{t} . \Delta n=0.01, \sigma=$ $2.0 \mu \mathrm{m}, x_{0}=10 \mu \mathrm{m}, H=34 \mu \mathrm{m}, \Delta n_{g}=0.001$. the two waveguide modes in the limit of long device lengths. In this case the system dynamics effectively reduce to three coupled modes and the coupled mode equations (9) can be solved analytically, as shown in the following.

Here we assume that the two waveguide modes have the same propagation constant $\left(\beta_{0}=\beta_{1}>0\right)$ and assign $\beta_{2}<0$ to the cladding mode. The waveguide modes are assumed to couple to the cladding mode with coupling coefficients $g e^{i \phi_{0}}$ and $g e^{i \phi_{1}}$ i.e., with the same amplitude but different phases. With the phase mismatch defined as $\Delta K=K_{g}-\beta_{0}+\beta_{2}$, Eq. (9) becomes

$$
\frac{d}{d z}\left[\begin{array}{l}
R_{0}(z) \\
R_{1}(z) \\
R_{2}(z)
\end{array}\right]=\frac{1}{2}\left[\begin{array}{ccc}
i \Delta K & 0 & -g \\
0 & i \Delta K & -g \\
-g & -g & -i \Delta K
\end{array}\right]\left[\begin{array}{l}
R_{0}(z) \\
R_{1}(z) \\
R_{2}(z)
\end{array}\right]
$$

where

$$
\begin{aligned}
& R_{0}(z)=A_{0}(z) e^{i \phi_{0}} e^{-\frac{i}{2} \Delta K z} \\
& R_{1}(z)=A_{1}(z) e^{i \phi_{1}} e^{-\frac{i}{2} \Delta K z} \\
& R_{2}(z)=A_{2}(z) e^{+\frac{i}{2} \Delta K z} .
\end{aligned}
$$

Note that the transformed amplitudes $R_{n}$ are defined slightly differently than $\mathcal{R}_{n}$ in Eq. (10). Upon diagonalizing the linear Eq. (11) the general solution can be written as a superposition of its eigensolutions,

$$
\begin{array}{r}
{\left[\begin{array}{l}
R_{0}(z) \\
R_{1}(z) \\
R_{2}(z)
\end{array}\right]=p_{0} e^{\frac{i}{2} \Delta K z}\left[\begin{array}{c}
-g \\
g \\
0
\end{array}\right]+q_{-} e^{-\gamma z}\left[\begin{array}{c}
g \\
g \\
i \Delta K+2 \gamma
\end{array}\right]} \\
+q_{+} e^{+\gamma z}\left[\begin{array}{c}
g \\
g \\
i \Delta K-2 \gamma
\end{array}\right]
\end{array}
$$

where $\gamma=\frac{1}{2} \sqrt{2 g^{2}-\Delta K^{2}}$ is one of the eigenvalues and $p_{0}, q_{-}$ and $q_{+}$are arbitrary constants. We apply the boundary conditions $R_{0}(z=0)=1, R_{1}(z=0)=0$ and $R_{2}(z=L)=0$ which corresponds to light at the input port of only one of the waveguides and no light launched into the cladding mode. This yields a linear set of equations that determine the values of $p_{0}, q_{-}$and $q+$ as

$$
\begin{aligned}
p_{0} & =-\frac{1}{2 g} \\
q_{+} & =\frac{Y^{2} \Delta K^{2}+2 \Delta K \Omega+4 X^{2} \Omega^{2}+i X Y\left[\Delta K^{2}-4 \Omega^{2}\right]}{g\left(Y^{2} \Delta K^{2}+4 X^{2} \Omega^{2}\right)} \\
q_{-} & =\frac{1}{2 g}-q_{+}
\end{aligned}
$$

where $X=\cos (\Omega L), Y=\sin (\Omega L)$.

We can distinguish two different regimes of behavior. Close to phase-matching, in the region where $|\Delta K| \leq \sqrt{2} g, \gamma$ is real and the last two terms of Eq. (13) exhibit exponential behavior. For a long device $(L \gg 1 / \gamma)$, we can easily show that the constant $q_{+} \rightarrow 0$ and so:

$$
\begin{aligned}
& R_{0}(z)=\frac{1}{2}\left(e^{i \Delta K z}+e^{-\gamma z}\right) \\
& R_{1}(z)=\frac{1}{2}\left(-e^{i \Delta K z}+e^{-\gamma z}\right) \\
& R_{2}(z)=\frac{1}{\sqrt{2}} e^{-\gamma z} .
\end{aligned}
$$


Thus, for $z \gg 1 / \gamma$ the power in the device (proportional to $\left|R_{n}\right|^{2}$ ) tends towards one quarter in each waveguide, the remaining half being reflected backwards into the cladding mode.

Outside of the bandgap (i.e. for $|\Delta K|>\sqrt{2} g$ ), all eigenvalues in Eq. (13) are imaginary and we have an oscillatory solution of the amplitudes of the light fields in the three modes. The three spatial eigenfrequencies are $\Delta K / 2$ and $\pm \Omega$ where $\Omega=$ $\frac{1}{2} \sqrt{\Delta K^{2}-2 g^{2}}=-i \gamma$. The propagating power in each mode is again given by $\left|A_{n}(z)\right|^{2}$. This results in a beating between the spatial oscillation frequencies, such that the power in the two waveguide modes has the following frequency terms: $2 \Omega$, $\Delta K / 2+\Omega, \Delta K / 2-\Omega$. A comparison of the analytic solutions (13) with a numerical solution of Eq. (10) including all cladding modes will be discussed in Sec. 5, as well as a more detailed investigation of the device behavior.

\section{PHASE-SELECTIVE GRATING INDUCED TRANS- PARENCY}

Equation (13) shows that our system allows for a specific superposition of light fields inside the two waveguides to be transmitted without loss through the device through grating induced transparency [14]. Specifically, light launched into the eigenvector

$$
\left[\begin{array}{c}
-g \\
g \\
0
\end{array}\right]
$$

i.e. in an antisymmetric superposition of light in the two waveguides, will be transmitted without ever coupling light into the cladding mode because of destructive interference of the coupling from the two individual waveguides. On the other hand, light launched into a symmetric superposition of fields in the two waveguides will couple strongly to the cladding mode and therefore, within the grating band gap $|\Delta K| \leq \sqrt{2} g$, will be totally reflected into the cladding mode. Thus, if light is launched simultaneously into both waveguides with the same amplitude the device can act as a phase-sensitive switch, i.e. transmitting or reflecting all light with $100 \%$ efficiency depending on the relative phase between the waveguides.

This result also explains the finding of Eq. (15) where one quarter of the light power, proportional to $\left|R_{n}\right|^{2}(n=0,1)$, is transmitted in each waveguide when light is launched into a single waveguide within the grating bandgap: Coupling light into a single waveguide at the input is equivalent to launching $50 \%$ into the symmetric and 50\% into the antisymmetric superposition mode of the two waveguides. Thus, $50 \%$ of the power is reflected into the backward propagating cladding mode and the remaining power is within the antisymmetric forward propagating waveguide superposition mode and therefore $25 \%$ of the power is in each waveguide.

We note that while in the discussion above we assumed equal coupling strengths of the two waveguides to the cladding mode, this is not in fact essential and an equivalent fully transmitted superposition mode exists also in the general case of the two waveguides coupling with different strengths and different relative phases to the cladding mode. In this case, the transmitted superposition mode contains unequal light power in the two waveguides.

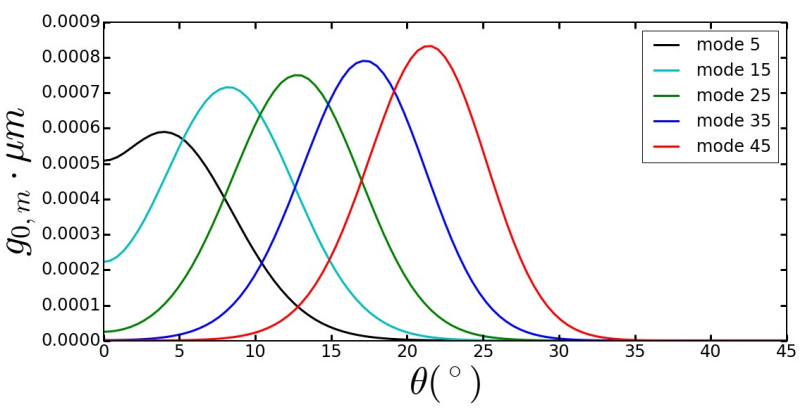

Fig. 5. Coupling coefficient between the core waveguide mode and a selection of backward propagating cladding modes as a function of tilt angle. $\Lambda_{g}=587.34 \mathrm{~nm}$ and $\Delta n_{g}=0.001$.

\section{NUMERICAL RESULTS}

For numerical simulations we pick a particular cladding mode to couple to the core modes. Specifically, in the following we choose mode number 35 with $n_{z}=1.1946$. The difference in $n_{z}$ from the nearest neighboring cladding mode is 0.0148 and thus the difference in propagation constant $0.0599 \mathrm{\mu m}^{-1}$ is larger than the coupling constants of order $10^{-3} \mu \mathrm{m}^{-1}$ for the parameters used below, which ensures that coupling of the waveguide modes will be predominantly with this one cladding mode for appropriately chosen grating periods. With $n_{z}=1.4445$ for the core waveguide modes, we find a grating period of $\Lambda_{g}=587.34 \mathrm{~nm}$ to achieve phase matching between the waveguide modes and this cladding mode. A grating depth of $\Delta n_{g}=0.001$ is used in the following [18].

In Fig. 5, we plot the coupling coefficient between the waveguide core mode and a selection of cladding modes, including the 35th mode, as a function of tilt angle as calculated from Eqs. (5)(7). We find a strong dependence on the tilt angle. In the vicinity of $\theta=0^{\circ}$ which corresponds to regular, non-tilted, Bragg gratings there is very little coupling between the waveguide core mode and the higher order cladding modes. Non-tilted gratings can therefore only be used to couple to some low order modes. However, the $n_{z}$ difference between low order modes is small, see Fig. 2, and therefore several cladding modes will be approximately phase matched with the grating simultaneously, thereby degrading the performance of the device. Reducing the grating strength will reduce this unwanted coupling to neighboring cladding modes but requires the use of unfeasible long devices.

Alternatively, higher order modes can be exploited which have much larger differences of $n_{z}$ between them and thus a single mode can be isolated to couple efficiently to the grating. To achieve strong coupling with realistic grating strengths and thus short device lengths tilted gratings are then required as shown in Fig. 5. This is the main driver for our device design based on tilted Bragg gratings and the use of higher order modes. The coupling coefficient with mode 35 is found to be greatest for a tilt angle of $17.23^{\circ}$.

For these parameters and a device length of $20 \mathrm{~mm}$, we show the evolution of the propagating power in the system as a function of distance in Fig. 6 obtained numerically including all cladding modes and compare the results with the analytic solution involving only three modes from Sec. 3. Here, we denote the waveguide that receives the input "waveguide 1 " and the other "waveguide 2".

In agreement with the discussion of Sec. 3, inside the bandgap, Fig. 6(a) shows the power asymptotically approach- 

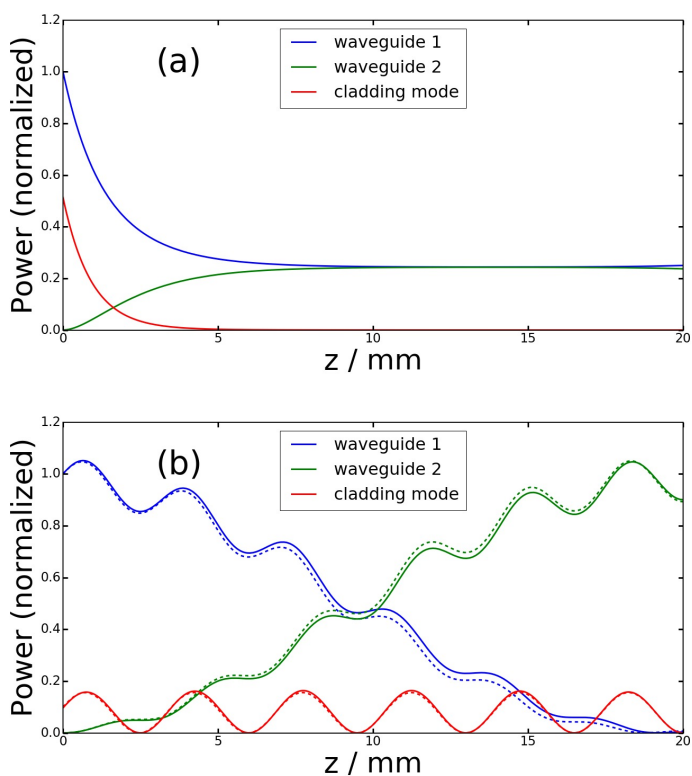

Fig. 6. Evolution of normalized propagating power in the two core waveguide modes and in the resonantly coupled cladding mode (35th mode) (a) inside the bandgap $\left(\lambda_{0}=1.55\right.$ $\mu \mathrm{m})$ and (b) outside the bandgap $\left(\lambda_{0}=1.54975 \mu \mathrm{m}\right)$. Solid curves are a numerical solution including all cladding modes, dashed curves are the analytical solution including only the 35th cladding mode. Parameters used are $\Lambda_{g}=587.34 \mathrm{~nm}$, $\theta=17.23^{\circ}$ and $\Delta n_{g}=0.001$.

ing one quarter of the input power in each waveguide with the remaining half back-reflected in the cladding mode. Outside the bandgap, Fig. 6(b), oscillatory behavior is found as expected. We chose the device length such that the majority of the light is coupled from one waveguide to the other, demonstrating its potential as a wavelength selective coupler. In both situations, the numerical results using the full set of cladding modes and the three-mode analytical solution using only the one cladding mode that is phase matched to the grating are in excellent agreement. Note that in Fig. 6(b), the power is normalized to the input power; as the core and cladding modes are counter-propagating, the device acts as a partial resonator and the local power in a mode can exceed unity. This shows that we can theoretically couple light between the two waveguides using the intermediary of one cladding mode without unwanted influence of other cladding modes. This justifies the analysis in Sec. 3 and allows us to greatly simplify the problem for further analysis.

In Fig. 7 we show how the output power in each of the two waveguides varies when light is launched into a single waveguide at the input as a function of wavelength. Close to the Bragg wavelength we find a photonic bandgap and the output power in both waveguides approaches one quarter of the input power, as discussed above. On either side of the bandgap we find two peaks where the maximum amount of power is transferred from one waveguide to the other. Far away from the bandgap, i.e., for a large phase mismatch, coupling between the waveguide and the cladding modes becomes inefficient and light is transmitted through the waveguide unchanged.

As the wavelength is varied, we can see oscillations in the output power of the waveguides. To understand these oscillations in more detail, we consider again the spatial oscilla-

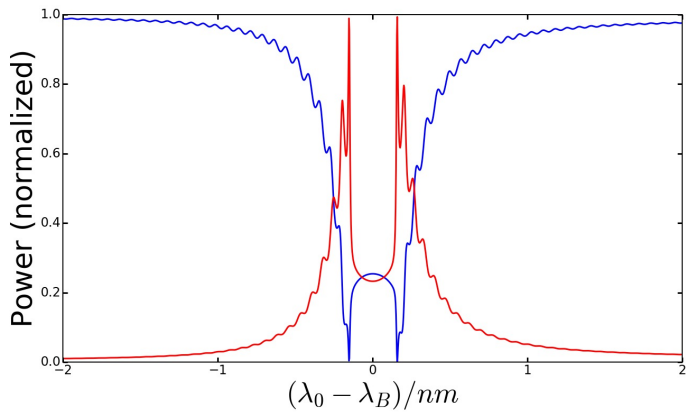

Fig. 7. Fractional output power from waveguides 1 (blue) and 2 (red) as a function of wavelength where at the input light is launched only into waveguide 1 . Parameters are $\Lambda_{g}=587.34$ $\mathrm{nm}, \theta=17.23^{\circ}, L=20 \mathrm{~mm}, \Delta n_{g}=0.001, \lambda_{B}=1.55 \mu \mathrm{m}$.

tion frequencies $2 \Omega, \Delta K / 2 \pm \Omega$ discussed in Sec. 3 (where $\Omega=\frac{1}{2} \sqrt{\Delta K^{2}-2 g^{2}}$ ) that describe the coupling of light between the waveguide modes and the selected cladding mode as a function of propagation distance. Away from the bandgap, where $\Delta K>\sqrt{2} g$, we have asymptotically $2 \Omega \approx \Delta K, \Delta K / 2+\Omega \approx \Delta K$ and $\Delta K / 2-\Omega \approx g^{2} /(2 \Delta K)$. There are thus two dominant spatial frequencies, a fast one scaling with $\Delta K$ and a slow one proportional to $1 / \Delta K$. As we can see from Fig. 6 , the fast oscillation describes power transfer between the waveguides and the cladding mode, whereas the slow oscillation describes power transfer between the two waveguides. At the output of the waveguides, Fig. 7, we thus see a slow variation of power transfer with a fast variation superimposed where power is lost into the backward propagating cladding mode.

This allows us to optimize the device to achieve a particular functionality. For example, if we want to achieve maximum power transfer from waveguide 1 to waveguide 2 at a given wavelength, we need to select a device length and a grating period (and thus $\Delta K$ ) such that simultaneously the slow oscillation leads to maximum power transfer at the device output while simultaneously the backscattered power in the cladding mode vanishes at $z=0$,

$$
\left(\frac{\Delta K}{2}-\Omega\right) L=\pi, \quad \Omega L=m \pi,
$$

where $m$ is an integer number; inserting Eqs. (16) into Eqs. (13)(14) confirms this.

The evolution of propagating light power for devices with $L$ and $\Delta K$ obtained by solving Eqs. (16) for $m=1,2,3$ is shown in Fig. 8 . In all three cases we find $100 \%$ power coupling from one waveguide to the other with 1,2 , and 3 complete oscillations of power into the cladding mode and back into the waveguides during propagation, respectively, as expected. This would allow our design to be used as a wavelength selective coupler e.g. for wavelength division multiplexing. Similar optimizations can also be performed to achieve other output power ratios, for example to form a wavelength selective 50-50 beamsplitter.

In order to assess the performance of our device as a frequency filter or wavelength division multiplexer we show in Fig. 9 the power output from the waveguides in the intermediate region between phase matching to two separate cladding modes. We choose the parameters $\Lambda_{g}=587.379 \mathrm{~nm}, L=9.739 \mathrm{~mm}$ which lead to $100 \%$ coupling at $\lambda_{0}=1.55 \mu \mathrm{m}$ as seen in Fig. 8 . Fig. 9 shows the resonances with modes 35 and 36 and a broad 

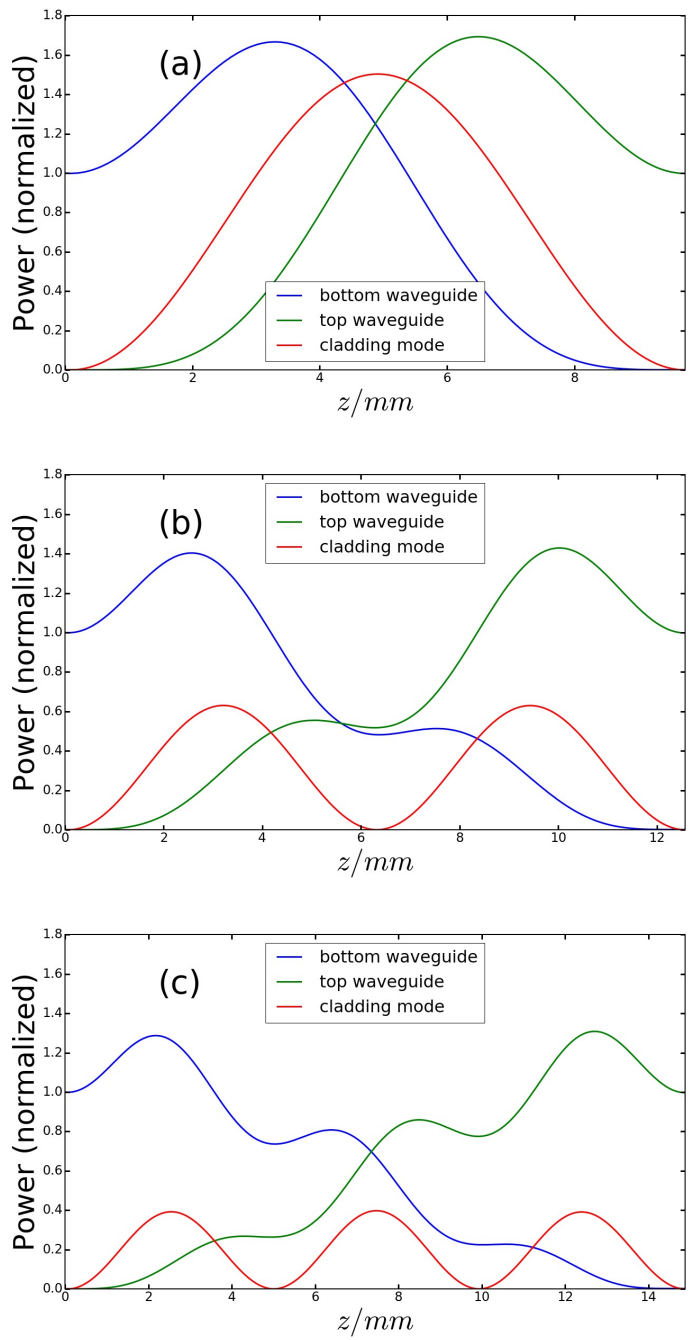

Fig. 8. Evolution of propagating power in the two waveguide modes and in the resonantly coupled cladding mode (35th mode) with parameters chosen to maximize power transfer between the waveguides with $m=1,2,3$ cladding mode oscillations. Parameters are (a) $\Lambda_{g}=587.379 \mathrm{~nm}, L=9.739$ $\mathrm{mm} \mathrm{(b)} \Lambda_{g}=587.391 \mathrm{~nm}, L=12.573 \mathrm{~mm}$ and (c) $\Lambda_{g}=$ $587.401 \mathrm{~nm}, L=14.876 \mathrm{~mm}$. In all cases $\theta=17.23^{\circ}$ and $\Delta n_{g}=0.001$.

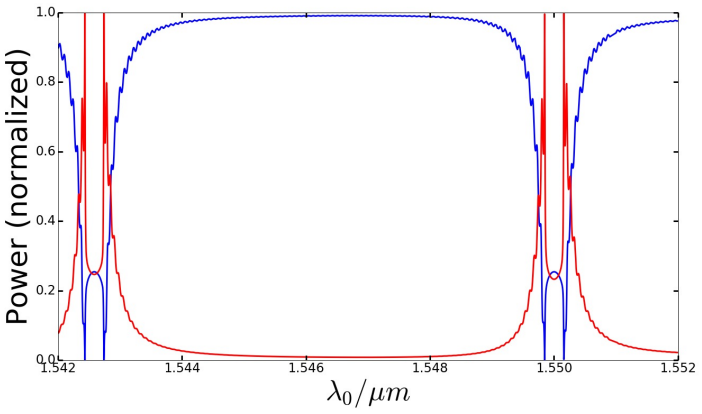

Fig. 9. Fractional output power from waveguides 1 (blue) and 2 (red) as a function of wavelength where at the input light is launched only into waveguide 1 . Parameters are $\Lambda_{g}=587.379$ $\mathrm{nm}, \theta=17.23^{\circ}, L=9.739 \mathrm{~mm}, \Delta n_{g}=0.001$.

wavelength region inbetween where the majority of light is transmitted in waveguide 1 and is not transferred to waveguide 2 by either of the cladding modes. At a wavelength of $1.5479 \mu \mathrm{m}$ we find a minimum transferred power of $0.80 \%$ which corresponds to an on-off ratio of $-21 \mathrm{~dB}$. Our device can therefore be used as a narrow-band frequency filter, for example, for separating a narrow-band signal from a pump in Raman or parametric amplifiers. However, the existence of closely spaced narrow resonances may require the use of additional broad-band filters in other applications such as wavelength division multiplexing.

\section{BANDWIDTH CONTROL}

In the previous section we presented a wavelength dependent waveguide coupler. In the following, we will now investigate the bandwidth of this device and its dependence on the device parameters. Here we define the bandwidth as the full width at half maximum of one of the two peaks shown in Fig. 7.

The dependence of the bandwidth on the device parameters is shown in Fig. 10 where we again utilize the 35th cladding mode as the intermediary between the two waveguide modes.

We can understand the results shown in Fig. 10 by deriving an approximate expression for the bandwidth in terms of the phase mismatch rather than the wavelength. The edges of the bandwidth correspond to where the device length is roughly one quarter and three quarters, on the outside and inside of the peak respectively, of the spatial oscillation period defined by the spatial frequency $\Delta K / 2-\Omega$, i.e. by replacing the right hand side of Eq. (16) with $\pi / 2$. Re-arranging this equation we find for the bandwidth

$$
\Delta K=\frac{L g^{2}}{3 \pi}-\frac{\pi}{2}
$$

For sufficiently long gratings, we thus expect the bandwidth of the device to scale linearly with the device length $L$ and quadratically with the coupling coefficient $g$. Since $g$ is proportional to the grating index contrast $\Delta n_{g}$, Eqs. (5)-(7), we thus expect the bandwidth to scale quadratically with $\Delta n_{g}$. This is in fact confirmed by the simulation results in Fig. 10. The dependence on the tilt angle can be explained in the same way by considering its effect on the coupling strength shown in Fig. 5.

For any practical device, the maximum grating index contrast is limited by the UV writing technique to about 0.001 , while short device lengths $(<10 \mathrm{~cm})$ [18] are generally desired to minimize scattering propagation losses. This suggests that changing 

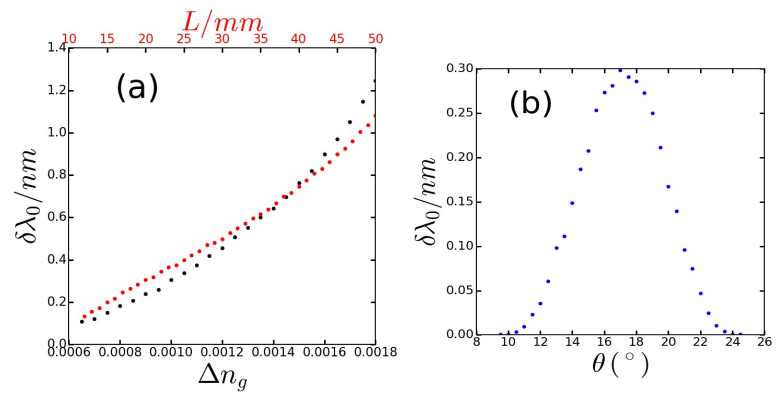

Fig. 10. Dependence of the bandwidth $\delta \lambda_{0}$ of the device as a wavelength-selective coupler as a function of (a) grating index contrast and device length, (b) grating tilt angle. Unless otherwise given, the parameters are $\Lambda_{g}=587.34 \mathrm{~nm}, L=$ $20 \mathrm{~mm}, \theta=17.23^{\circ}$, and $\Delta n_{g}=0.001$.

the tilt angle is an interesting option to control the device bandwidth.

The bandwidth of our device is around $0.3 \mathrm{~nm}$ for parameters of $\Lambda_{g}=587.34 \mathrm{~nm}, L=20 \mathrm{~mm}, \theta=17.23^{\circ}$, and $\Delta n_{g}=0.001$. This narrow bandwidth is caused by our choice of using a counter-propagating cladding mode as intermediary between the two waveguide modes, which leads to a phase mismatch of $\Delta K=K_{g}-\left|\beta_{0}\right|-\left|\beta_{2}\right|$ between waveguide and cladding modes that is highly sensitive to changes of the operating wavelength. The bandwidth is much wider if a co-propagating cladding mode (and correspondingly long period gratings) are selected for the device operation [24].

\section{THERMAL STABILITY}

In this section we explore the effect of temperature on the modal structure of the device and thus the wavelength of operation. The temperature affects the device in two different ways: through the temperature dependence of the refractive index, and through the change of device dimensions because of thermal expansion. We model the first effect using the three term temperature-dependent Sellmeier equation [27] for silica to provide the index of the cladding. We assume for simplicity that the index contrast of the waveguide and of the grating do not depend on temperature. The thermal expansion of silica at room temperature is assumed linear with $\alpha_{L}=5.5 \times 10^{-7} \mathrm{~K}^{-1}$ [28]. We scale all dimension of our device (cladding width, waveguide width, waveguide seperation, grating period and device length) linearly with temperature for a device with $H=34 \mu \mathrm{m}$, $x_{0}=10 \mu \mathrm{m}, \sigma=2 \mu \mathrm{m}, \Lambda_{g}=585.068 \mathrm{~nm}$ and $L=20 \mathrm{~mm}$ that is phase matched at room temperature $(T=300 \mathrm{~K})$.

For these parameters we find that the propagation constants of the waveguide and resonant (35th) cladding mode change by $3.38 \times 10^{-5} \mathrm{~m}^{-1} /{ }^{\circ} \mathrm{C}$ and $4.13 \times 10^{-5} \mathrm{~m}^{-1} /{ }^{\circ} \mathrm{C}$, respectively, while the grating wave vector $K_{g}$ changes by $-5.91 \times 10^{-6}$ $\mathrm{m}^{-1} /{ }^{\circ} \mathrm{C}$. We see that the largest contributions to the phase mismatch come from the changes in propagation constants of the modes and not from the shift in grating period due to thermal expansion. The changes in propagation constants are themselves largely due to the change in refractive index of the device and not thermal expansion.

Overall, this leads to a change of resonant wavelength by 11.7 $\mathrm{pm} /{ }^{\circ} \mathrm{C}$, which is consistent with the shift reported elsewhere for Bragg gratings in the same material [29]. Figure (11) shows

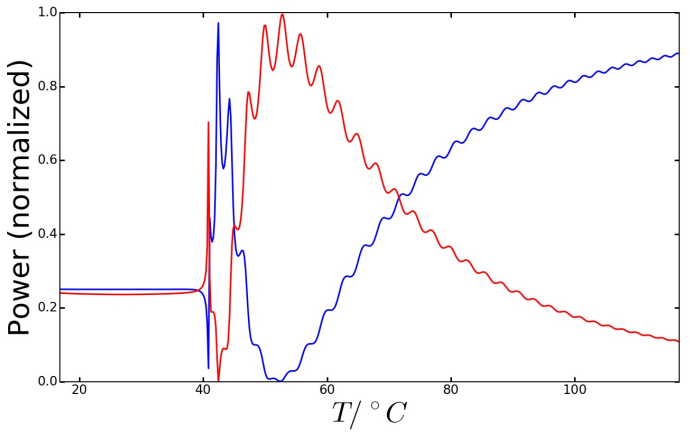

Fig. 11. Fractional output power from waveguides 1 (blue) and 2 (red) as a function of temperature where at the input light is launched only into waveguide 1 . Parameters are $\Lambda_{g}=$ $587.34 \mathrm{~nm}, \theta=17.23^{\circ}, L=20 \mathrm{~mm}, \Delta n_{g}=0.001, \lambda_{0}=1.55 \mu \mathrm{m}$.

the device output as a function of temperature for a launched wavelength of $1550 \mathrm{~nm}$. We note that a temperature change of approximately $30^{\circ} \mathrm{C}$ is required to shift the resonance curve by one bandwidth, which implies that the device is highly robust to temperature fluctuations.

\section{CONCLUSIONS}

We have proposed and investigated theoretically and numerically a new type of integrated waveguide coupler based on UV written tilted Bragg gratings. A ridge structure is exploited to tailor the cladding modes of the device which act as intermediaries to couple light between the waveguides. Using realistic parameters, we demonstrate that by introducing a tilt angle to the Bragg grating planes light can be efficiently coupled between the two waveguides using only a single cladding mode as an intermediary. This simplifies the system to an effective threemode device which allows us to achieve a near lossless coupler with a very narrow bandwith, high on-off ratio and high thermal stability. Our numerical simulations are supported by simplified analytical estimates which can be used to easily design the device functionality, for example, by controlling the operation bandwidth of the waveguide coupler.

The device has a wide range of potential applications in classical telecommunications and in quantum technology. Specifically, in this work we demonstrated applications as a waveguide coupler with arbitrary coupling efficiency up to $100 \%$, as a narrowband coupler and/or wavelength filter, and as a phase-sensitive switch.

\section{FUNDING}

EPSRC Quantum Technology Programme (EP/M013243/1, $\mathrm{EP} / \mathrm{M} 013294 / 1)$.

\section{DATA AVAILABILITY}

The data used in this article is openly available at [30].

\section{REFERENCES}

1. A. K. Ghatak and K. Thyagarajan, Introduction to fiber optics (Cambridge University Press, New Dehli, 1999).

2. D. Marcuse, "Directional Couplers Made of Nonidentical Asymmetric Slabs. Part II: Grating-Assisted Couplers," J. Light. Technol. 5, 268-273 (1987). 
3. J. L. Archambault, P. S. Russell, S. Barcelos, P. Hua, and L. Reekie, "Grating-frustrated coupler: a novel channel-dropping filter in singlemode optical fiber." Opt. Lett. 19, 180 (1994).

4. D. Mechin, P. Grosso, and D. Bosc, "Add - Drop Multiplexer With UVWritten Bragg Gratings and Directional Coupler in SiO 2 - Si Integrated Waveguides," J. Light. Technol. 19, 1282-1286 (2001).

5. K. Ikeda, M. Nezhad, and Y. Fainman, "Wavelength selective coupler with vertical gratings on silicon chip," Appl. Phys. Lett. 92, 201111 (2008).

6. D. T. H. Tan, K. Ikeda, S. Zamek, A. Mizrahi, M. P. Nezhad, A. V. Krishnamoorthy, K. Raj, J. E. Cunningham, X. Zheng, I. Shubin, Y. Luo, and $Y$. Fainman, "Wide bandwidth, low loss 1 by 4 wavelength division multiplexer on silicon for optical interconnects," Opt. Express 19, 24012409 (2011).

7. W. Shi, H. Yun, C. Lin, M. Greenberg, X. Wang, Y. Wang, S. T. Fard, J. Flueckiger, N. A. F. Jaeger, and L. Chrostowski, "Ultra-compact, flat-top demultiplexer using anti-reflection contra-directional couplers for cwdm networks on silicon," Opt. Express 21, 6733-6738 (2013).

8. T. Erdogan and J. Sipe, "Tilted fiber phase gratings," J. Opt. Soc. Am. A 13, 296-313 (1996).

9. K. S. Lee, "Mode coupling in tilted planar waveguide gratings," Appl. Opt. 39, 6144-6149 (2000)

10. C. K. Madsen, J. Wagener, T. A. Strasser, D. Muehlner, M. A. Milbrodt, E. J. Laskowski, and J. DeMarco, "Planar waveguide optical spectrum analyzer using a UV-induced grating," IEEE J. Sel. Top. Quantum Electron. 4, 925-929 (1998).

11. C. Riziotis and M. N. Zervas, "Design considerations in optical add/drop multiplexers based on grating-assisted null couplers," J. Light. Technol. 19, 92-104 (2001).

12. K. K. Mehta and R. J. Ram, "Precise and diffraction-limited waveguideto-free-space focusing gratings," Sci. Rep. 7, 2019 (2017).

13. C. Holmes, L. G. Carpenter, H. L. Rogers, I. J. G. Sparrow, J. C. Gates, and P. G. R. Smith, "Planar waveguide tilted Bragg grating refractometer fabricated through physical micromachining and direct UV writing," Opt. Express 19, 12462-8 (2011).

14. H.-C. Liu and A. Yariv, "Grating induced transparency (GIT) and the dark mode in optical waveguides," Opt. Express 17, 11710-11718 (2008).

15. B. J. Metcalf, N. Thomas-Peter, J. B. Spring, D. Kundys, M. A. Broome, H. P. C., X.-M. Jin, M. Barbieri, W. S. Kolthammer, J. C. Gates, B. J. Smith, N. K. Langford, P. G. R. Smith, and I. A. Walmsley, "Multiphoton quantum interference in a multiport integrated photonic device," Nat. Commun. 4 (2013). 1356

16. J. Carolan, C. Harrold, C. Sparrow, E. Martín-López, N. J. Russell, J. W. Silverstone, P. J. Shadbolt, N. Matsuda, M. Oguma, M. Itoh, G. D. Marshall, M. G. Thompson, J. C. F. Matthews, T. Hashimoto, J. L. O'Brien, and A. Laing, "Universal linear optics," Science 349, 711-716 (2015).

17. O. Alibart, J. Fulconis, G. K. L. Wong, S. G. Murdoch, W. J. Wadsworth, and J. G. Rarity, "Photon pair generation using four-wave mixing in a microstructured fibre: theory versus experiment," New J. Phys. 8 (2006). 67.

18. C. Sima, J. C. Gates, H. L. Rogers, P. L. Mennea, C. Holmes, M. N. Zervas, and P. G. R. Smith, "Ultra-wide detuning planar Bragg grating fabrication technique based on direct UV grating writing with electrooptic phase modulation." Opt. Express 21, 15747-15754 (2013).

19. C. Sima, "Integrated Planar Bragg Grating Devices for Advanced Optical Communication Systems," Ph.D. thesis, University of Southampton (2013).

20. M. T. Posner, P. L. Mennea, N. Podoliak, P. Horak, J. C. Gates, and P. G. R. Smith, " $45^{\circ}$ tilted gratings for silica based integrated polarizers," in The European Conference on Lasers and Electro-Optics, (Munich, Germany, 2015).

21. M. T. Posner, P. L. Mennea, N. Podoliak, P. Horak, J. C. Gates, and P. G. R. Smith, "Integrated polarizing coupler based on tilted gratings," in Integrated Photonics Research, Silicon and Nanophotonics, (Vancoover, Canada, 2016).

22. Y. Bai and K. S. Chiang, "Analysis and Design of Long-Period
Waveguide-Grating Couplers," J. Light. Technol. 23, 4363-4373 (2005).

23. Y. Bai, Q. Liu, K. P. Lor, and K. S. Chiang, "Widely tunable long-period waveguide grating couplers," Opt. Express 14, 2595-2597 (2006).

24. K. C. Chow, K. S. Chiang, Q. Liu, K. P. Lor, and H. P. Chan, "UVwritten long-period waveguide grating coupler for broadband add/drop multiplexing," Opt. Commun. 282, 378-381 (2009).

25. C. Holmes, K. R. Daly, I. J. G. Sparrow, J. C. Gates, G. D’Alessandro, and P. G. R. Smith, "Excitation of Surface Plasmons Using Tilted Planar-Waveguide Bragg Gratings," IEEE Photonics J. 3, 777-788 (2011).

26. B. Fornberg, "Generation of Finite Difference Formulas on Arbitrarily Spaced Grids," Math. Comput. 51, 699-706 (1988).

27. D. B. Leviton and B. J. Frey, "Temperature-dependent absolute refractive index measurements of synthetic fused silica," in Optomechanical Technologies for Astronomy, (Orlando, United States, 2006).

28. Accuratus, "Fused Silica, $\mathrm{SiO} 2$ Glass Properties," (https://www.accuratus.com/fused.html (accessed 28/03/2019)).

29. C. Holmes, D. Kundys, J. Gates, C. Gawith, and P. Smith, " $150 \mathrm{GHz}$ of thermo-optic tuning in direct UV written silica-on-silicon planar Bragg grating," Electron. Lett. 45, 954 (2009).

30. M. J. Weisen, M. T. Posner, J. C. Gates, C. B. E. Gawith, P. G. R. Smith, and P. Horak, "Low loss wavelength-selective integrated waveguide coupler based on tilted bragg gratings (dataset)," (University of Southampton, 2019, https://doi.org/10.5258/SOTON/D0870). 